\title{
Spatio-Temporal Distribution of Malaria in Yunnan Province, China
}

\author{
Feng-Ming Hui, Bing Xu, Zhang-Wei Chen, Xiao Cheng, Lu Liang, Hua-Bing Huang, Li-Qun Fang, Hong Yang, \\ Hong-Ning Zhou, Heng-Lin Yang, Xiao-Nong Zhou, Wu-Chun Cao,* and Peng Gong* \\ State Key Laboratory of Remote Sensing Science, Jointly Sponsored by the Institute of Remote Sensing Applications of Chinese Academy of \\ Sciences and Beijing Normal University, Beijing, People's Republic of China; College of Global Change and Earth System Science, Beijing \\ Normal University, Beijing, People's Republic of China; State Key Laboratory of Pathogen and Biosecurity, Beijing Institute of Microbiology \\ and Epidemiology, Beijing, People's Republic of China; Department of Environmental Science and Engineering, Tsinghua University, \\ Beijing, People's Republic of China; Yunnan Institute of Parasitic Diseases, Pu'er, People's Republic of China; National Institute \\ of Parasitic Diseases, Chinese Center for Disease Control and Prevention, Shanghai, People's Republic of China
}

\begin{abstract}
The spatio-temporal distribution pattern of malaria in Yunnan Province, China was studied using a geographic information system technique. Both descriptive and temporal scan statistics revealed seasonal fluctuation in malaria incidences in Yunnan Province with only one peak during 1995-2000, and two apparent peaks from 2001 to 2005. Spatial autocorrelation analysis indicated that malaria incidence was not randomly distributed in the province. Further analysis using spatial scan statistics discovered that the high risk areas were mainly clustered at the bordering areas with Myanmar and Laos, and in Yuanjiang River Basin. There were obvious associations between Plasmodium vivax and Plasmodoium falciparum malaria incidences and climatic factors with a clear 1-month lagged effect, especially in cluster areas. All these could provide information on where and when malaria prevention and control measures would be applied. These findings imply that countermeasures should target high risk areas at suitable times, when climatic factors facilitate the transmission of malaria.
\end{abstract}

\section{INTRODUCTION}

Malaria is one of the main arthropod-borne infectious diseases that are seriously harmful to human health in mainland China. The report of the National Surveillance Project indicated that there were over 740 thousand malaria cases diagnosed in 907 counties of 18 provinces in 2003. ${ }^{1}$ Yunnan Province is one of the most severe epidemic regions, and ranked first for the number of reported cases and second for the incident rate of the disease from 1999 to $2004 .^{2}$ The persistence and re-emergence of malaria in Yunnan Province have been attributed to importing of infective sources from countries of southeastern Asia in bordering areas, increase in floating population, and wide distribution of multiple vectors with high density around the year. ${ }^{3}$ However, the mechanisms of the persistence and spread of either Plasmodium vivax or Plasmodium falciparum malaria in the province are unclear. Both high risk areas and high risk periods of the disease have not been identified. The lack of this knowledge has prohibited us from understanding the transmission dynamics of malaria in this region, and improving intervention strategies and control measures.

The emergence and development of spatial epidemiology have recently provided us a useful approach to understanding spatial patterns of disease and identifying risk factors. ${ }^{4,5}$ A geographic information system (GIS) and spatial statistics such as spatial autocorrelation, spatial scan, and variograms were previously applied to recognize malaria hotspot areas, ${ }^{6}$ determine the spatial and temporal dynamics, ${ }^{7}$ find risk factors, ${ }^{8}$ and predict malaria transmission risk. ${ }^{9}$ In addition, the environmental factors associated with disease vectors could be mapped and analyzed using GIS techniques, which were applied for surveillance of vectors and quantitative assess-

*Address correspondence to Wu-Chun Cao, State Key Laboratory of Pathogen and Biosecurity, Beijing Institute of Microbiology and Epidemiology, Beijing 100071, P.R. China, E-mail: caowc@nic.bmi.ac .cn and Peng Gong, State Key Laboratory of Remote Sensing Science, Jointly Sponsored by the Institute of Remote Sensing Applications of Chinese Academy of Sciences and Beijing Normal University, Beijing 100101, P.R. China, E-mail: gong@nature.berkeley.edu. ment of transmission risk of malaria. ${ }^{10-12}$ In this study, the temporal and spatial analyses of both $P$. vivax and $P$. falciparum malaria were conducted using GIS techniques to understand the trend and dynamics of transmission, to identify risk periods and areas, and to determine environmental factors associated with increased risk of malaria.

\section{MATERIALS AND METHODS}

Study area. Yunnan Province is situated at $21^{\circ} 08^{\prime}-$ $29^{\circ} 16^{\prime}$ north latitude and $97^{\circ} 31^{\prime}-106^{\circ} 12^{\prime}$ east longitude of southwestern China, and borders Myanmar in the west and Laos and Vietnam in the south (Figure 1). The province has an area of $394,000 \mathrm{~km}^{2}$ and population of 45 million. The seasonal average temperature ranges from 10 to $15^{\circ} \mathrm{C}$. The diurnal difference temperature usually fluctuates from 12 to $20^{\circ} \mathrm{C}$. The annual rainfall is approximately $1,100 \mathrm{~mm}$, about $85 \%$ of which occurs between May and October. Six water systems with more than 600 rivers run through the province. ${ }^{13}$ The diverse geographic landscapes and complex climate situations provide favorable breeding sites for Anopheles mosquitoes, the vector of malaria.

Data collection and management. Data on malaria were obtained from Yunnan Institute of Parasitic Diseases (YIPD), where a passive surveillance was conducted. The physicians working at hospitals and clinics are required to report suspected patients to YIPD. The malaria cases were defined based on the diagnostic criteria of the World Health Organization (WHO). ${ }^{14}$

Demographic data at the county level were obtained from the Chinese Natural Resources Database. ${ }^{15}$ The annual malaria incidence was calculated by using the annual population of each county. It was assumed that each resident in the county was at risk for infection of malaria. Monthly malaria incidences of the province were calculated to understand seasonal fluctuation of malaria incidence. Malaria cases and incidence of each county were coded according to geographic location (geo-coded) and matched to the corresponding polygon on a digital map of Yunnan Province using ArcGIS 9.2 (ESRI Inc., Redlands, CA). 


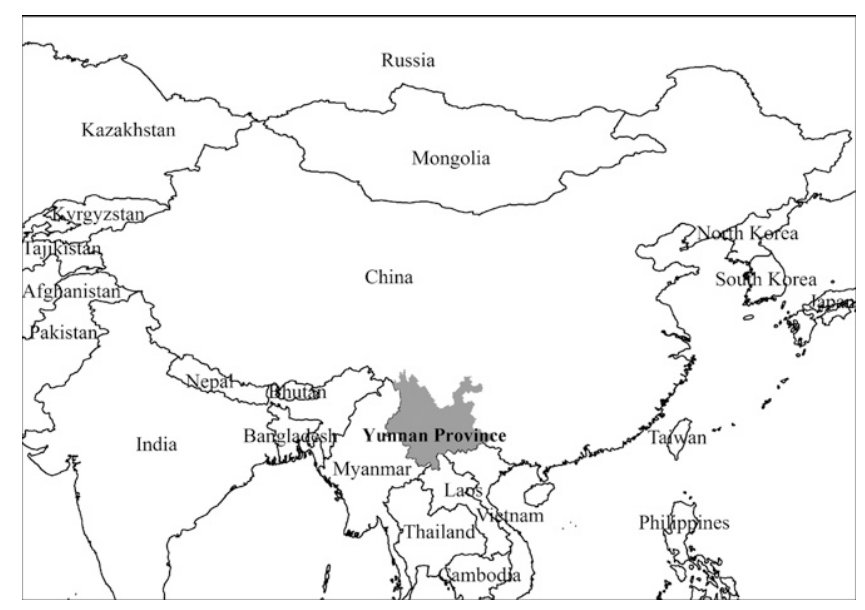

Figure 1. Location of Yunnan Province in China.

Monthly climate data from 1995 to 2005 in Yunnan Province were obtained from China Meteorological Data Sharing Service System, ${ }^{16}$ which contained information on rainfall, average temperature (AT), average maximum temperature (MaxT), average minimum temperature (MinT), and relative humidity $(\mathrm{H})$. All of them were collected from 32 weather stations in the province.

Temporal distribution analysis. The malaria incidences in Yunnan Province were plotted by year and by month to observe the annual and seasonal fluctuation. In addition to the descriptive approach to the temporal data, an analytic approach was carried out to determine the temporal cluster quantitatively. Temporal scan statistics were performed to detect temporal clusters of malaria cases using SaTScan7.03 software. ${ }^{17}$ The "temporal scan statistics" were applied to test the null hypothesis that the incidence of malaria was randomly distributed. To avoid preselection bias, varied time periods were scanned without knowledge on time cluster size. The SaTScan software set circular windows of various sizes, and moved each window over the time. Whenever the window encountered a new case, a likelihood function was calculated to test for elevated risk within the window in comparison with those outside the window. The likelihood function for any given window was proportional to

$$
\left(\frac{n}{\mu}\right)^{n}\left(\frac{N-n}{N-\mu}\right)^{N-n} I()
$$

where $N$ is the total number of cases, $n$ is the observed number of cases within the window, and $\mu$ is the expected number of cases within the window. The indicator function $I()$ is a binary validation element that allows the likelihood function to detect clusters of high values $[I(n>\mu)]$, low values $[I(n<\mu)]$, or both $[I()=1] \cdot{ }^{17,18}$ In this study, the time aggregation length was set to 1 month and the maximum temporal cluster size was set to the default value $\leq 50 \%$ of the study period. For each window of varying size, the software tested the risk of malaria within and outside the window, with the null hypothesis of equal risk.

Spatial autocorrelation analysis. Global Moran's I was calculated for $P$. vivax malaria and $P$. falciparum malaria incidence in each year in GeoDa0.9.5-i software. ${ }^{19}$ First, a contiguity-based spatial weight was constructed for each county by creating a queen contiguity weights file. Queen contiguity uses all common points (boundaries and vertices) to define a neighbor, which means two counties are neighbors even if there is only one common point. In a contiguity-based spatial weight file the weights $\left\{w_{i j}\right\}$ could be defined as follows:

$$
w_{i j}= \begin{cases}1 & \text { region } i \text { and region } j \text { are contiguous } \\ 0 & \text { otherwise }\end{cases}
$$

Spatial autocorrelation for malaria incidence was calculated based on the assumption of constant variance. This assumption was usually violated when incidences at county level varied greatly in different populations. The empirical Bayes transformation was performed to adjust for the violation of the assumption. $^{20,21}$ In GeoDa0.9.5-i software, malaria cases, population, and the spatial weight file were entered into the empirical Bayes model to smooth the malaria incidence. In fact, Moran's $I$ calculated by spatial empirical Bayes model is much more powerful than that directly by the rate. ${ }^{21}$ And then Moran's $I$ scatter plot was produced with a spatial lag of incidence on the vertical axis and a standardized incidence on the horizontal axis. Any observation beyond the two standard deviations was categorized as outliers. Finally, a significance test was performed through the permutation test, and a reference distribution was generated under an assumption that the incidence was randomly distributed. The number of permutation tests was set to 999 and the significance level was set as 0.001. In this study, Moran's $I$ values of $P$. vivax and $P$. falciparum malaria were calculated using annual incidences for each county.

Spatial cluster analysis. Spatial cluster analysis was performed to detect spatial clusters or hotpots of malaria using SaTScan7.03 software. ${ }^{17}$ The analysis process of spatial scan is similar to that of temporal scan. The SaTScan imposed circular windows of varying size on the spatial data. The likelihood of malaria within or outside the window was calculated; a relative risk (RR) was created and the statistical significance was assessed using a Monte Carlo simulation, which is carried out under the null hypothesis of random distribution. The window was moved over the study region and centered on the centroid of each county polygon. The area within the window varied in size from zero to some upper limit (a maximum radius of the circular window set in virtue of the proportion of the whole population), never including $>50 \%$ of the total population. Whenever the window encountered a new case, the likelihood function (Equation 1) was calculated to test for elevated risk within the window compared with those outside the window. In this study, the maximum window radius was set to be smaller than $20 \%$ of the total population. For each window of varying position and size, the software tested the risk of malaria within and outside the window, with the null hypothesis of equal risk.

Spearman correlation analysis. Spearman correlation analysis was conducted to examine the association between monthly malaria incidence and climate variables in the whole province as well as the spatial cluster areas using SPSS software (version 13.0, SPSS Inc., Chicago, IL).

\section{RESULTS}

Temporal variation in malaria incidence. The annual incidence of $P$. vivax malaria in Yunnan Province varied from 


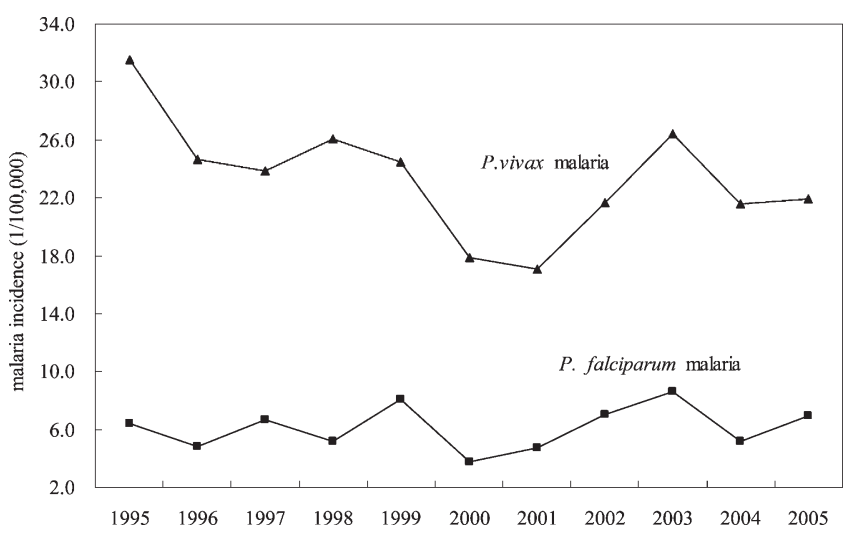

FIGURE 2. Annual incidences of Plasmodium vivax and Plasmodium falciparum malaria from 1995 to 2005 in Yunnan Province, China.

1995 to 2005 (Figure 2), with a lowest incidence of 17.07 per 100,000 people in 2001, and a highest incidence of 31.53 per 100,000 people in 1995. Although the incidences of $P$. falciparum malaria were usually $3 \sim 5$ times lower than those of $P$. vivax malaria in each corresponding year, the variation in annual incidence of the disease was essentially consistent with that of $P$. vivax malaria (Figure 2). The lowest incidence of $P$. falciparum malaria was observed in 2000 , and the highest in 2003. Although malaria cases occurred in every month of the year, the seasonal effect in the incidence of malaria in Yunnan Province was obvious, with summer and autumn as peak times. From 1995 to 2000, basically only one peak was observed, starting generally from June, reaching a peak in July and August, and ending in September (Figure 3A), when more
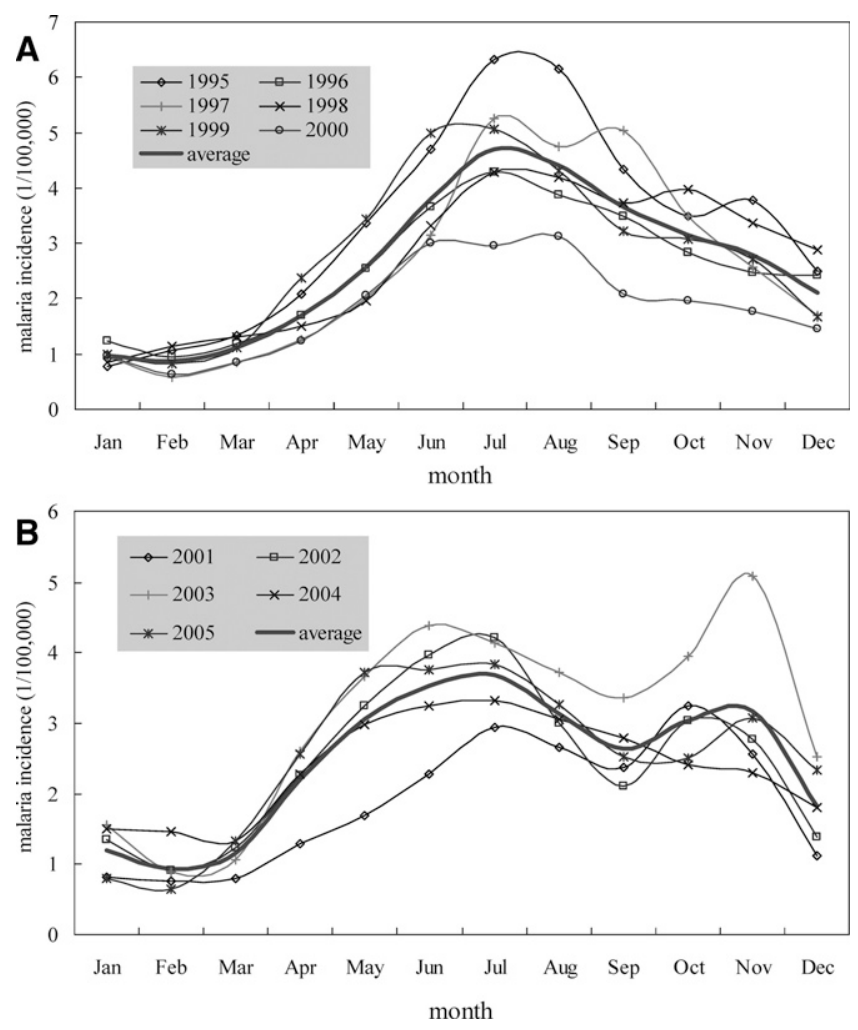

FIGURE 3. Monthly incidences of malaria in Yunnan Province, China in different time phases.
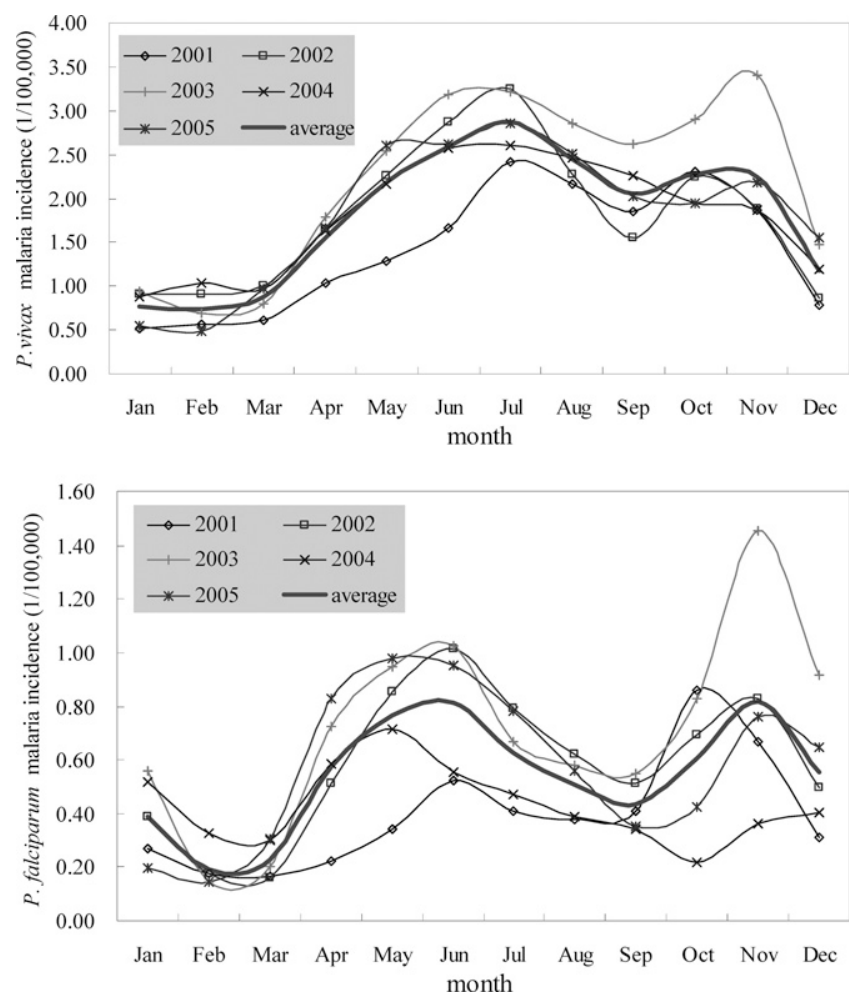

Figure 4. Monthly incidences of Plasmodium vivax and Plasmodium falciparum malaria during 2001-2005 in Yunnan Province, China.

than half of the malaria cases were reported. Since 2001, there had usually been two incident peaks, one of which occurred from June to July and the other from October to November (Figure 3B). There was also a remarkable difference in monthly peak between $P$. vivax and $P$. falciparum malaria during the years 2001-2005 (Figure 4). The incident peak of $P$. falciparum malaria was much clearer, and the first peak occurred a month earlier than that of $P$. vivax malaria.

The temporal clusters of $P$. vivax and $P$. falciparum malaria. To understand the temporal variation quantitatively, temporal scan statistics were performed, using 1 month as the aggregation length and the maximum temporal cluster size of $\leq 50 \%$ of the study period. The temporal clusters were identified and showed in Table 1 . The temporal clusters determined by using temporal scan statistics analysis were highly consistent with the identified peaks through the descriptive approach by plotting the monthly incidence of malaria. The quantitative analyses not only further testified that the incident peak of $P$. falciparum malaria usually occurred (in April) 1 month earlier than P. vivax malaria in Yunnan Province, but also differentiate the principal incident peak (the most likely temporal cluster) of the two (Table 1).

Spatial autocorrelation of $\boldsymbol{P}$. vivax and $\boldsymbol{P}$. falciparum malaria. Global spatial autocorrelation was carried out using GeoDa0.9.5-i software. ${ }^{19}$ The spatial autocorrelation analysis for annualized incidence of malaria in Yunnan Province showed that the Moran's I values were statistically significant in most years except for P. vivax in 1999 and 2000 (Table 2). The findings implied that malaria distribution was generally autocorrelated in space in the province. The Moran's $I$ values for P. vivax malaria increased from 1995 to 1997, then decreased until 2000, and dramatically increased afterward. The Moran's 
TABLE 1

Temporal clusters of malaria cases from 1995 to 2005 in Yunnan Province

\begin{tabular}{|c|c|c|c|c|c|c|c|c|c|}
\hline & \multicolumn{3}{|c|}{ Malaria incidence } & \multicolumn{3}{|c|}{ Plasmodium vivax malaria incidence } & \multicolumn{3}{|c|}{ Plasmodium falciparum malaria incidence } \\
\hline & Cluster period & Relative risk & $P$ & Cluster period & Relative risk & $P$ & Cluster period & Relative risk & $P$ \\
\hline 1995 & Jun-Nov & 2.572 & 0.001 & - & - & - & - & - & - \\
\hline 1996 & Jun-Oct & 2.024 & 0.001 & - & - & - & - & - & - \\
\hline 1997 & Jun-Nov & 3.29 & 0.001 & - & - & - & - & - & - \\
\hline 1998 & Jun-Nov & 2.353 & 0.001 & - & - & - & - & - & - \\
\hline 1999 & May-Oct & 2.448 & 0.001 & - & - & - & - & - & - \\
\hline 2000 & May-Oct & 2.175 & 0.001 & - & - & - & - & - & - \\
\hline 2001 & Jun-Nov & 2.266 & 0.001 & Jun-Nov & 2.593 & 0.001 & Oct-Nov & 2.375 & 0.001 \\
\hline 2002 & May-Oct & 1.645 & 0.001 & May-Oct & 1.964 & 0.001 & May-July & 1.794 & 0.001 \\
\hline 2003 & Jun-Nov & 1.989 & 0.001 & Jun-Nov & 2.197 & 0.001 & Nov & 2.275 & 0.001 \\
\hline 2004 & May-Oct & 1.652 & 0.001 & May-Oct & 1.833 & 0.001 & Apr-Jun & 1.682 & 0.001 \\
\hline 2005 & Apr-Sep & 1.834 & 0.001 & May-Oct & 1.939 & 0.001 & Apr-Sep & 2.082 & 0.001 \\
\hline
\end{tabular}

$I$ values for $P$. falciparum malaria were remarkably fluctuant and generally higher than those of $P$. vivax malaria, indicating that the $P$. falciparum malaria epidemic area was more focalized than $P$. vivax malaria.

The spatial clusters of $\boldsymbol{P}$. vivax and $\boldsymbol{P}$. falciparum malaria. Using the maximum spatial cluster size of $\leq 20 \%$ of the total population, $P$. vivax and $P$. falciparum malaria spatial clusters were identified (Figure 5). Considering the difference in incident peaks of malaria in Yunnan Province, the data of 1995-2000 and 2001-2005 were analyzed, respectively.

Using the data of 1995-2000, the spatial scan statistics identified a most likely cluster encompassed 22 counties mainly in the southwest of Yunnan Province, which included 13.1\% of the total population. The overall RR within the cluster was $9.63(P<0.001)$, with an observed number of cases of 42,237 compared with 9,336 expected cases. The secondary clusters included 12 counties with $6.6 \%$ of the population (Figure 5 ). When the data of 2001-2005 were used for the analysis, a most likely cluster of 9 counties covering $5.34 \%$ of the total population was discovered and the overall RR of the cluster was $13.36(P<0.001)$. Interestingly, the 9 counties with the most likely cluster were out of the 12 counties identified as the secondary cluster in the previous analysis of the data from 1995 to 2000. The secondary clusters encompassed 24 counties, 21 of which were counties identified as the most likely cluster by the previous analysis of the data from 1995 to 2000 .

The spatial scan statistics analysis of the data from 1995 to 2000 identified a most likely cluster in 6 counties bordering Myanmar, which had $4.06 \%$ of the total population. The over-

TABLE 2

Moran's I values for Plasmodium vivax and Plasmodium falciparum malaria from 1995 to 2005 in Yunnan Province

\begin{tabular}{|c|c|c|c|c|c|c|}
\hline \multirow[b]{2}{*}{ Year } & \multicolumn{3}{|c|}{ P. vivax malaria } & \multicolumn{3}{|c|}{ P. falciparum malaria } \\
\hline & $\begin{array}{l}\text { Incident rate } \\
(1 / 100,000)\end{array}$ & Moran's I & $P$ & $\begin{array}{l}\text { Incident rate } \\
(1 / 100,000)\end{array}$ & Moran's I & $P$ \\
\hline 1995 & 31.534 & 0.2765 & 0.012 & 6.386 & 0.5539 & 0.001 \\
\hline 1996 & 24.688 & 0.2835 & 0.009 & 4.828 & 0.2560 & 0.012 \\
\hline 1997 & 23.832 & 0.3621 & 0.002 & 6.657 & 0.4005 & 0.003 \\
\hline 1998 & 26.098 & 0.2143 & 0.028 & 5.193 & 0.3155 & 0.005 \\
\hline 1999 & 24.490 & 0.1720 & 0.116 & 8.120 & 0.5002 & 0.002 \\
\hline 2000 & 17.887 & 0.1344 & 0.173 & 3.755 & 0.4211 & 0.003 \\
\hline 2001 & 17.070 & 0.3988 & 0.002 & 4.739 & 0.3803 & 0.003 \\
\hline 2002 & 21.668 & 0.4591 & 0.003 & 7.056 & 0.4814 & 0.001 \\
\hline 2003 & 26.432 & 0.4708 & 0.004 & 8.602 & 0.6091 & 0.001 \\
\hline 2004 & 21.585 & 0.4753 & 0.003 & 5.178 & 0.5505 & 0.001 \\
\hline 2005 & 21.943 & 0.5855 & 0.001 & 6.934 & 0.4624 & 0.002 \\
\hline
\end{tabular}

all RR was $15.38(P<0.001)$. The secondary cluster encompassing 6 counties was within the southeast and west of the province bordering Laos and Vietnam, with $6.69 \%$ of the total population. The overall RR within the clusters was 8.81 $(P<0.001)$. When analyzed with the data from 2001 to 2005, two more counties bordering Myanmar were added to the most likely cluster except for the former 6 counties. The most likely cluster had $4.96 \%$ of the total population with the overall RR of 31.55 $(P<0.001)$. Eleven counties were included in the secondary cluster, which was remarkably different from the results of the former analysis. Seven counties within the cluster were in the southwest of the province bordering Laos and Myanmar, and 4 counties were within the Yuanjiang River Basin mainly bordering Vietnam.

Correlation between monthly malaria incidence and climatic varibles. Spearman correlation analyses were performed relating monthly malaria incidence to monthly climatic variables (Table 3 ). The monthly AT, monthly average MaxT and MinT, monthly amount of rainfall, and monthly average relative $\mathrm{H}$ were significantly positive-correlated with monthly malaria incidences over the study period (Table 3). The correlation coefficients between monthly incidence and climatic variables for $P$. vivax malaria were greater than those for $P$. falciparum malaria (Table 3). Among the climatic variables, the monthly average minimum temperature was most closely correlated to both $P$. vivax and $P$. falciparum malaria incidence. Considering the possible lagged effect of climatic variables on the occurrence of malaria, the correlations between malaria incidences and the previous climatic variables of the previous month were estimated. The correlation coefficients between monthly incidences and AT, MaxT, MinT in the previous month $\left(\mathrm{AT}_{1}, \mathrm{MaxT}_{1}, \mathrm{MinT}_{1}\right)$ were greater than those in the same month (Table 3 ) for both $P$. vivax and $P$. falciparum malaria, indicating an obvious 1-month lagged effect. The correlation coefficient between monthly incidence and average rainfall in the previous month seemed unchanged when the data of the same month were used. The relative humidity in the previous month had little or no effect on malaria incidence (Table 3).

To understand the possible spatial differences in association between malaria incidence and climate factors, spearman correlation analyses were carried out in the cluster area and non-cluster area (Table 4). For P. vivax malaria, the correlation coefficients between monthly incidence and AT, MaxT, MinT, and rainfall in the same or previous month were remarkably greater in cluster areas than those in either non-cluster areas or the whole province. For P. falciparum malaria, no obvious 

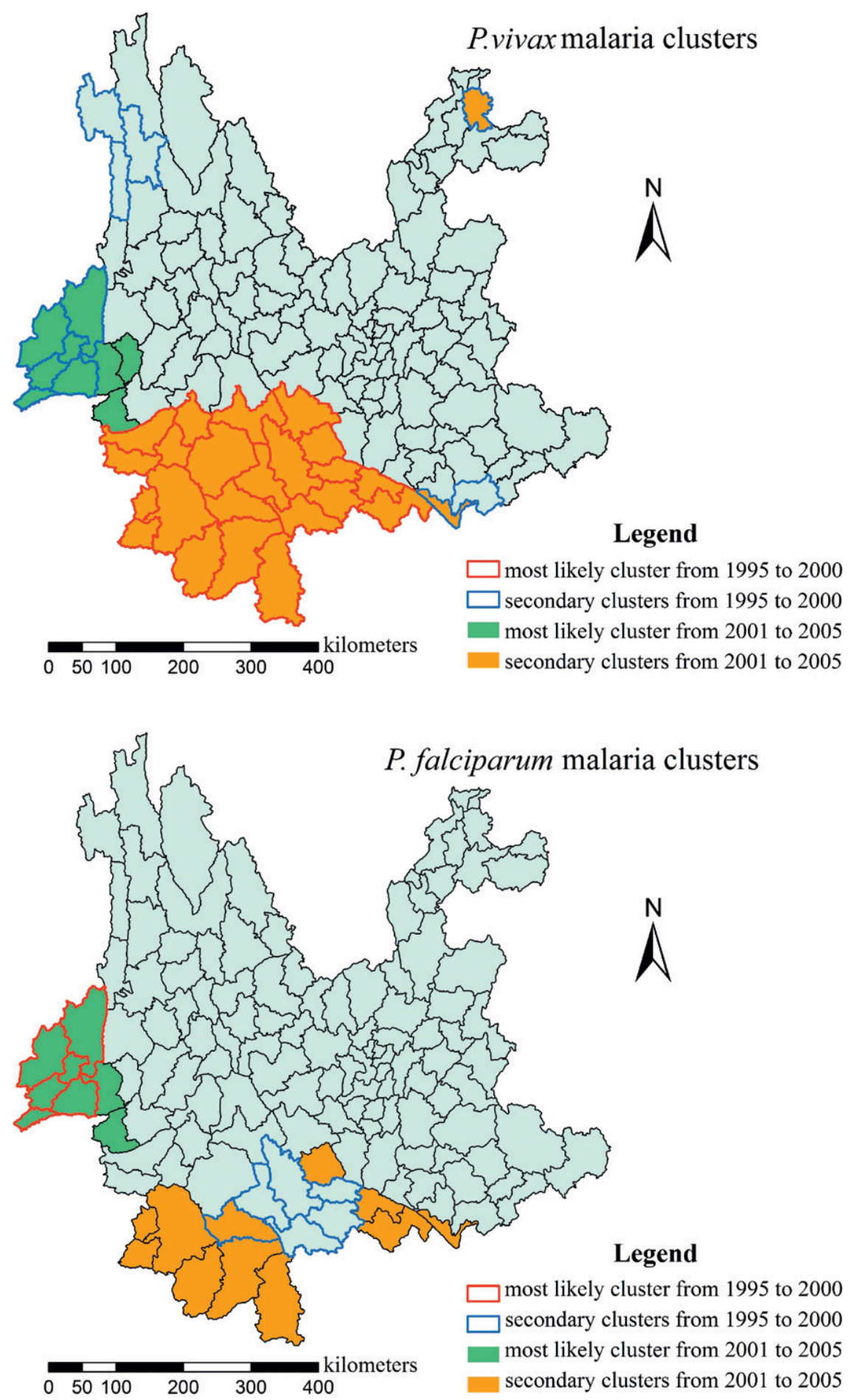

FIgURE 5. Spatial distribution of identified clusters of Plasmodium vivax and Plasmodium falciparum malaria cases with significantly higher incidence rates using the maximum cluster size $\leq 20 \%$ of the total population in Yunnan, 1995-2005.

change was observed in correlation coefficients in cluster and non-cluster areas.

\section{DISCUSSION}

In this study, we found that the malaria incidence in Yunnan Province had not substantially changed during the 11-year period from 1995 to 2005 (Figure 2), although intensive intervention had been carried out there. The findings imply that malaria remains a serious health threat in the province. The variations in annual incidences of both $P$. vivax and $P$. falciparum malaria basically followed the same patterns indicating that certain factors rather than biologic characteristics of parasites determine the transmission of the disease. ${ }^{3}$ Since 1995 , social-economic activities in Yunnan Province have greatly changed. Many factors, like exploiture in the river valley region (a torrid zone with altitude below $800 \mathrm{~m}$ ), constructions of national highways and expressways, and trade development 
TABLE 3

Spearman correlation results of malaria incidence and climate factors*

\begin{tabular}{|c|c|c|c|c|c|c|}
\hline & \multicolumn{2}{|c|}{ Malaria incidence } & \multicolumn{2}{|c|}{$\begin{array}{l}\text { Plasmodium vivax } \\
\text { malaria incidence }\end{array}$} & \multicolumn{2}{|c|}{$\begin{array}{l}\text { Plasmodium falciparum } \\
\text { malaria incidence }\end{array}$} \\
\hline & $\mathrm{r}$ & $P$ & $\mathrm{r}$ & $P$ & $\mathrm{r}$ & $P$ \\
\hline $\mathrm{AT}_{0}$ & 0.710 & $<0.001$ & 0.771 & $<0.001$ & 0.387 & $<0.001$ \\
\hline $\operatorname{MaxT}_{0}$ & 0.599 & $<0.001$ & 0.676 & $<0.001$ & 0.332 & 0.004 \\
\hline $\operatorname{MinT}_{0}$ & 0.761 & $<0.001$ & 0.795 & $<0.001$ & 0.384 & $<0.001$ \\
\hline Rainfall $_{0}$ & 0.692 & $<0.001$ & 0.685 & $<0.001$ & 0.356 & 0.002 \\
\hline $\mathrm{H}_{0}$ & 0.723 & $<0.001$ & 0.641 & $<0.001$ & 0.315 & 0.007 \\
\hline $\mathrm{AT}_{1}^{0}$ & 0.826 & $<0.001$ & 0.824 & $<0.001$ & 0.426 & $<0.001$ \\
\hline $\mathrm{MaxT}_{1}$ & 0.797 & $<0.001$ & 0.804 & $<0.001$ & 0.438 & $<0.001$ \\
\hline $\operatorname{MinT}_{1}^{1}$ & 0.818 & $<0.001$ & 0.806 & $<0.001$ & 0.397 & $<0.001$ \\
\hline Rainfall $_{1}$ & 0.702 & $<0.001$ & 0.689 & $<0.001$ & 0.290 & 0.014 \\
\hline $\mathrm{H}_{1}$ & 0.445 & $<0.001$ & 0.351 & 0.003 & 0.073 & 0.541 \\
\hline
\end{tabular}

*AT $=$ monthly average temperature; MaxT $=$ monthly average maximum temperature; MinT $=$ monthly average minimum temperature; Rainfall $=$ monthly average amount of precipitation; $\mathrm{H}=$ monthly average relative humidity. The subscript numbers: $0=$ the current month climate factors; 1 = the previous 1 month climate factors.

especially in the border areas had accelerated population movement, which might have facilitated malaria transmission.

Both descriptive and temporal scan statistics revealed seasonal fluctuations in malaria incidence (Figure 3, Table 1). There was only one peak from 1995 to 2000 and two apparent peaks since 2001 (Figure 4). The peak times of malaria occurred in summer and autumn when farmers usually worked in the field and slept in the open. This situation made residual spraying ineffective and thus facilitated malaria transmission. These findings imply that countermeasures should be opportunely taken, and focused on increasing knowledge of malaria prevention and encouraging farmers to use mosquito nets. At the same time, the temporal fluctuation in malaria incidence was closely related to agricultural activities. For instance, farmers cultivate crops in summer and harvest in autumn, when mosquitoes propagate actively because of feasible climate conditions. These activities often increase the opportunities of mosquito biting, and consequently increase the risk of human infection. In addition, many other factors possibly contribute to the seasonal variations, such as climatic changes, ecological and environmental determinates. Further studies are required to study the role of these variables in malaria transmission in the region.

Spatial autocorrelation analysis revealed that malaria was not randomly distributed in Yunnan Province. Spatial scan further confirmed that malaria cases clustered in the province. The high risk areas were mainly in the borders with Myanmar and Laos, and in Yuanjiang River Basin. However, the primary and secondary clusters varied regarding different types of malaria and different periods of time. The analyses using the GIS technique and temporal and spatial statistics could study the temporal and spatial distribution of malaria and identify times and areas of increased risk in Yunnan Province, where both $P$. vivax and $P$. falciparum malaria are prevalent. These findings imply that suitable countermeasures should target high risk areas accordingly. Further studies are required to study the underlying causes of increased risk in the identified areas.

Many factors, such as vector species and abundance, human behavior, population immunity, social and economic status, and control measures, are known to have significant influence on the transmission of malaria. Climatic variables are considered as the environmental factors for increased risk of malaria because of their impacts on the Plasmodium incubation rate and mosquito vector activities. To understand the contribution of climatic factors to the transmission of malaria in Yunnan Province, spearman correlation analyses between malaria incidence and climatic variables were conducted. Among the climatic factors investigated in the study, temperature was the most influencing factor, especially with a onemonth lag effect. It is understandable that there was usually an approximate 1-month period in the course of the malaria infection cycle from wiggler becoming an infectious mosquito to mosquito biting humans and finally human developing malaria symptoms. It also implies that malaria prevalence is sensitive to temperature changes. Malaria prevention and control agencies, therefore, should pay great attention to temperature changes. In contrast, the correlation coefficient for the association between monthly malaria incidence and synchronomous relative humidity was greater than that for the association between monthly malaria incidence rate and relative humidity in the previous month. This suggests that relative humidity only influences the activities of mosquitoes. Rainfall showed complex association with malaria incidence. The rainfall often leads to small puddles serving as mosquito breeding sites and increases humidity, which enhances mosquito survival. However, the relationship between mosquito abundance and rainfall was nonlinear. Abundant rainfall and accumulation of surface water in complicated terrain probably wash out or destroy mosquito breeding sites, which may subsequently reduce mosquito density. A certain amount of rainfall may also prevent people from working outdoors resulting in fewer chances of mosquito bites and consequently decrease the malaria incidence. Findings of spearman correlation analyses in the cluster area and non-cluster area indicate that there

TABLe 4

Spearman correlation results of malaria incidence and climate factors in cluster and non-cluster areas*

\begin{tabular}{|c|c|c|c|c|c|c|c|c|}
\hline & \multicolumn{4}{|c|}{ Plasmodium vivax malaria incidence } & \multicolumn{4}{|c|}{ Plasmodium falciparum malaria incidence } \\
\hline & \multicolumn{2}{|c|}{ Cluster area } & \multicolumn{2}{|c|}{ Non-cluster area } & \multicolumn{2}{|c|}{ Cluster area } & \multicolumn{2}{|c|}{ Non-cluster area } \\
\hline & $\mathrm{r}$ & $P$ & $\mathrm{r}$ & $P$ & $\mathrm{r}$ & $P$ & $\mathrm{r}$ & $P$ \\
\hline $\mathrm{AT}_{0}$ & 0.852 & $<0.001$ & 0.619 & $<0.001$ & 0.401 & 0.002 & 0.393 & 0.002 \\
\hline $\operatorname{MaxT}_{0}$ & 0.806 & $<0.001$ & 0.389 & 0.002 & 0.260 & 0.045 & 0.333 & 0.009 \\
\hline $\operatorname{MinT}_{0}^{0}$ & 0.873 & $<0.001$ & 0.707 & $<0.001$ & 0.461 & $<0.001$ & 0.403 & 0.001 \\
\hline Rainfall $_{0}$ & 0.774 & $<0.001$ & 0.597 & $<0.001$ & 0.411 & 0.001 & 0.318 & 0.013 \\
\hline $\mathrm{H}_{0}$ & 0.677 & $<0.001$ & 0.655 & $<0.001$ & 0.450 & $<0.001$ & 0.461 & $<0.001$ \\
\hline $\mathrm{AT}^{0}$ & 0.860 & $<0.001$ & 0.812 & $<0.001$ & 0.545 & $<0.001$ & 0.545 & $<0.001$ \\
\hline $\mathrm{MaxT}_{1}$ & 0.854 & $<0.001$ & 0.725 & $<0.001$ & 0.543 & $<0.001$ & 0.512 & $<0.001$ \\
\hline $\operatorname{MinT}_{1}$ & 0.839 & $<0.001$ & 0.810 & $<0.001$ & 0.532 & $<0.001$ & 0.541 & $<0.001$ \\
\hline Rainfall $_{1}$ & 0.782 & $<0.001$ & 0.680 & $<0.001$ & 0.455 & $<0.001$ & 0.444 & $<0.001$ \\
\hline $\mathrm{H}_{1}$ & 0.360 & 0.005 & 0.435 & $<0.001$ & -0.006 & 0.966 & 0.321 & 0.012 \\
\hline
\end{tabular}

*AT $=$ monthly average temperature; MaxT $=$ monthly average maximum temperature; MinT $=$ monthly average minimum temperature; Rainfall $=$ monthly average amount of precipitation; $\mathrm{H}=$ monthly average relative humidity. The subscript numbers: $0=$ the current month climate factors; $1=$ the previous 1 month climate factors. 
are spatial differences of the influence of climate variables on malaria risk, especially for $P$. vivax malaria there are greater spatial differences.

Malaria epidemic patterns in Yunnan have changed. The epidemics of $P$. vivax and $P$. falciparum malaria are different. Climate factors are highly influencing malaria transmission. More detailed and other environmental data should be used in future research. Spatial clusters of $P$. vivax and $P$. falciparum malaria changed with time, which were mainly located in bordering regions and the Yuanjiang River Basin. These findings should be considered in future malaria prevention and control projects and in the development of spatio-temporal models of malaria transmission in Yunnan as well.

Received September 13, 2008. Accepted for publication May 20, 2009.

Acknowledgments: We thank two anonymous reviewers for valuable comments and suggestions.

Financial support: This work was supported by the National Natural Science Foundation of China (No. 30590370), the National High Technology Research and Development Program of China (2006AA12Z112), the National Science Fund for Distinguished Young Scientists (No. 30725032), and Special Grant for the Prevention and Control of Infectious Diseases (No. 2008ZX10004-012).

Authors' addresses: Feng-Ming Hui, State Key Laboratory of Remote Sensing Science, Jointly Sponsored by the Institute of Remote Sensing Applications of Chinese Academy of Sciences and Beijing Normal University, Beijing 100101, P.R. China, College of Global Change and Earth System Science, Beijing Normal University, Beijing 100875, P.R. China, and State Key Laboratory of Pathogen and Biosecurity, Beijing Institute of Microbiology and Epidemiology, Beijing 100071, P.R. China. Bing Xu, Department of Environmental Science and Engineering, Tsinghua University, Beijing 100084, P.R. China. ZhangWei Chen, Hong-Ning Zhou, and Heng-Lin Yang, Yunnan Institute of Parasitic Diseases, Pu'er 665000, P.R. China. Xiao Cheng, College of Global Change and Earth System Science, Beijing Normal University, Beijing 100875, P.R. China. Lu Liang, Hua-Bing Huang, and Peng Gong, State Key Laboratory of Remote Sensing Science, Jointly Sponsored by the Institute of Remote Sensing Applications of Chinese Academy of Sciences and Beijing Normal University, Beijing 100101, P.R. China. Li-Qun Fang, Hong Yang, and Wu-Chun Cao, State Key Laboratory of Pathogen and Biosecurity, Beijing Institute of Microbiology and Epidemiology, Beijing 100071, P.R. China. Xiao-Nong Zhou, National Institute of Parasitic Diseases, Chinese Center for Disease Control and Prevention, Shanghai 200025, P.R. China.

\section{REFERENCES}

1. Ministry of Health, 2005. Malaria Surveillance Project in China.

2. Hui FM, 2007. A spatio-temporal analysis on the patterns of malaria epidemic in Yunnan Province using GIS [dissertation]. Nanjing: Nanjing University, 29-30.
3. $\mathrm{Li} \mathrm{ZH,2006.} \mathrm{Analysis} \mathrm{of} \mathrm{main} \mathrm{factors} \mathrm{associated} \mathrm{with} \mathrm{the} \mathrm{preva-}$ lence of malaria in Yunnan Province at present. China Trop Med 6: 1383-1384.

4. Elliott P, Wartenberg D, 2004. Spatial epidemiology: current approaches and future challenges. Environ Health Perspect 112: 998-1006.

5. Ostfeld RS, Glass GE, Keesing F, 2005. Spatial epidemiology: an emerging (or re-emerging) discipline. Trends Ecol Evol 20: 328-335.

6. Ernst KC, Adoka SO, Kowuor DO, Wilson ML, John CC, 2006. Malaria hotspot areas in a highland Kenya site are consistent in epidemic and non-epidemic years and are associated with ecological factors. Malar J 5: 78.

7. Zhou GF, Sirichaisinthop J, Sattabongkot J, Jones J, Bjornstad ON, Yan GY, Cui LW, 2005. Spatio-temporal distribution of Plasmodium falciparum and P. vivax malaria in Thailand. Am J Trop Med Hyg 72: 256-262.

8. Kleinschmidt I, Sharp BL, Clarke GPY, Curtis B, Fraser C, 2001. Use of generalized linear mixed models in the spatial analysis of small-area malaria incidence rates in KwaZulu Natal, South Africa. Am J Epidemiol 153: 1213-1221.

9. Kleinschmidt I, Bagayoko M, Clarke GP, Craig M, Le Sueur D, 2000. A spatial statistical approach to malaria mapping. Int $J$ Epidemiol 29: 355-361.

10. Zhou GF, Minakawa N, Githeko A, Yan GY, 2004. Spatial distribution patterns of malaria vectors and sample size determination in spatially heterogeneous environments: a case study in the west Kenyan highland. J Med Entomol 41: 1001-1009.

11. Zhou GF, Munga S, Minakawa N, Githeko A, Yan GY,2007. Spatial relationship between adult malaria vector abundance and environmental factors in Western Kenya Highlands. Am J Trop Med Hyg 77: 29-35.

12. Poncon N, Tran A, Toty C, Luty AJF, Fontenille D, 2008. A quantitative risk assessment approach for mosquito-borne diseases: malaria re-emergence in southern France. Malar J 7: 147.

13. Introductions of Yunnan Province, 2008. Available at: http://www .yn.gov.cn/yunnan, china/74590868828323840/index.html. Accessed August 1, 2008.

14. WHO, 1998. WHO Expert Committee on Malaria: Twentieth Report. (WHO Technical Report Series No.892), 18-24.

15. Chinese Natural Resources Database, 2008. Available at: http:// www.naturalresources.csdb.cn/index.asp. Accessed August 1, 2008.

16. China Meteorological Data Sharing Service System, 2008. Available at: http://cdc.cma.gov.cn. Accessed August 1, 2008.

17. Kulldorff M, 2006. SaTScan User Guide for vesion 7.0. Available at: http://www.satscan.org/. Accessed August 1, 2008.

18. Kulldorff M, Heffernan R, Hartman J, Assun ão R, Mostashari F, 2005. A space-time permutation scan statistic for the early detection of disease outbreaks. PLoS Med 2: 216-224.

19. Ansenlin L, 2003. GeoDa 0.9 User's Guide: Spatial Analysis Laboratory (SAL). Department of Agricultural and Consumer Economics, University of Illinois, Urbana-Champaign, IL.

20. Meza JL, 2003. Empirical Bayes estimation smoothing of relative risks in disease mapping. J Stat Plan Infer 112: 43-62.

21. Assuncao RM, Reis EA, 1999. A new proposal to adjust Moran's I for population density. Stat Med 18: 2147-2162. 